\title{
Félix Bouvier \\ Histoire du Séminaire de Mont-Laurier. Formation d'une élite et d'une classe moyenne.
}

\author{
Montréal : Éditions Fides, 2005, 267 pages.
}

\section{Michel Aubin}

Ce livre est une version de la thèse de doctorat de l'auteur soutenue en 2003 à la Faculté des sciences de l'éducation de l'Université de Montréal. L'auteur a voulu, selon ses propres mots, " mettre en lumière le rôle joué par le Séminaire de Mont-Laurier entre 1910 et 1965 dans la formation d'une élite et d'une classe moyenne, et cela, dans le contexte d'une région de colonisation de la deuxième demie du XIX ${ }^{\mathrm{e}}$ siècle et des sept premières décennies du XXe ${ }^{\mathrm{e}}$ (p. 7).

Lauteur a divisé son livre en trois grandes parties. La première présente le contexte précédant et entourant la fondation et l'histoire du séminaire. Il s'agit d'abord, dans le chapitre 1, de l'enseignement classique dans le milieu canadien-français, dans lequel s'insérera le Séminaire de Mont-Laurier, dont le but est de former, surtout sur le plan moral, une élite religieuse et nationale.

Le chapitre 2 est particulièrement important et intéressant en ce qu'il situe l'étude dans son cadre historiographique. L'auteur y traite entre autres de la colonisation périlaurentienne, la situant dans le contexte plus large du surpeuplement de la plaine du Saint-Laurent, de l'émigration aux États-Unis, de la faible industrialisation au Québec et, enfin, de la crainte suscitée chez le clergé catholique par l'exode rural et l'émigration. Se référant à l'œuvre de Gérard Bouchard, dont il juge le modèle historiographique " particulièrement novateur" (p. 45), l'auteur situe toutefois son étude dans une perspective différente : alors que Bouchard insiste sur l'histoire sociale de la classe paysanne d'une région de colonisation, il vise plutôt l'étude de l'élite d'une telle région qui a été formée dans un séminaire d'enseignement classique traditionnel (p. 45). En ce qui concerne l'idéologie entourant la colonisation, il se réfere particulièrement aux études de Gabriel Dussault et de Christian Morrissonneau dont il reprend les concepts de messianisme, de mythe et d'utopie, d'ordre religieux et national (p. 46-52) Le projet 
et le discours du curé Labelle, figure emblématique de la colonisation du Nord, représentent bien cette idéologie. À cet effet, il est important de souligner que son projet de colonisation ne se limitait pas à l'agriculture, comme on a longtemps prétendu, mais proposait plutôt le développement intégral de la région, tant sur les plans du commerce et de l'industrie que sur celui de l'agriculture. L'auteur cherche donc à montrer le rôle d'une institution d'enseignement classique, offrant également un cours commercial et située dans une région nouvelle, dans la formation d'une élite en ces différents domaines. Enfin, le troisième chapitre traite de l'évolution socioreligieuse du Québec et des Hautes-Laurentides de la fin du 19e siècle aux années 1960.

Mettant largement à contribution les archives du Séminaire de Mont-Laurier, dont des entretiens avec d'anciens élèves, l'auteur, dans la deuxième partie du livre, retrace l'histoire du Séminaire de Mont-Laurier depuis la demande du curé Labelle en 1879 jusqu'à sa fermeture en 1965, en passant par la formation de la Corporation du Collège de Nominingue en 1881, la fondation de ce même collège en 1910, son transfert vers Mont-Laurier et sa transformation en Séminaire Saint-Joseph de Mont-Laurier en 1915 suite à la création du diocèse de Mont-Laurier. La contribution du Collège SainteMarie-de-Monnoir (1853-1912) est soulignée par l'emprunt que fait le Séminaire au programme de Monnoir, notamment la présence d'un cours commercial, et par l'apport de ses prêtres lorsqu'il fermera ses portes en 1912. On retrouve donc dans cette partie toute la vie de ce collège-séminaire à travers des thèmes comme le difficile recrutement du personnel, les constructions à Mont-Laurier, les activités des élèves, les constitutions définissant son organisation et ses objectifs, et les changements de programmes. Selon l'auteur, le cours commercial et le cours classique avaient des objectifs complémentaires : former une élite par le cours classique et une classe moyenne par le cours commercial. La culture humaniste acquise chez la classe moyenne devait permettre à celle-ci d'être l'intermédiaire entre l'élite et la masse de la population (p.107). L'auteur passe également en revue les activités religieuses, en analysant leur rôle d'encadrement. Il fait de même pour les activités d'éducation sociale comme la Jeunesse étudiante catholique, introduite dans les années 1930 et fort active jusqu'à la fermeture du collège, ainsi que pour les activités de formation nationale à travers la fête annuelle de Dollard, considéré à l'époque comme le héros national par excellence, et les débats oratoires. Les activités sportives du collège sont aussi présentées.

Les années 1950-1965 commencent dans le même esprit que les décennies précédentes, soit la formation d'une élite cléricale d'abord, laïque ensuite, dans un fort esprit religieux et nationaliste canadien-français. Le Séminaire devient exclusivement classique, une école de métiers et une école d'agriculture étant maintenant autonomes. Mais un nouveau contexte s'implante graduellement : baisse des vocations sacerdotales, scolarisation secondaire plus recherchée, pression pour que l'institution réponde à la demande d'une scolarisation générale plus élevée, augmentation du nombre d'enseignants laïques et syndicalisation de ces derniers, augmentation des coûts conduisant à des déficits, attitudes et attentes différentes des élèves. La mutation socioreligieuse québécoise s'affirme à Mont-Laurier. Le Séminaire continue d'insister sur sa vocation régionale et sur les valeurs religieuses et nationalistes. Cependant, 
l'intervention de la Faculté des arts et de l'État en faveur de changements se faisant de plus en plus pressante, $\mathrm{M}^{\mathrm{gr}}$ André Ouellet, lors d'une réunion avec le Conseil du Séminaire en 1964, pose carrément la question de la vente de l'institution. Ce sera fait en 1965. La région perd alors la formation post-secondaire.

La dernière partie du livre est une étude statistique dont le but est de vérifier si le Séminaire a permis la production et/ou la reproduction des rapports sociaux dans la région et de comparer avec d'autres études faites sur les séminaires de Chicoutimi, Trois-Rivières et Nicolet ou encore celle faite pour l'ensemble des collèges-séminaires par Claude Galarneau. L'auteur doit cependant se concentrer sur une courte période, soit celle de 1919-1938, faute de données pour les autres périodes. Il réussit à couvrir les questions qu'il avait posées et à comparer son étude aux autres. Il fait ressortir certains traits caractéristiques de l'institution tels la présence significative d'élèves venant d'en dehors de la région/diocèse, notamment des États-Unis, l'origine des prêtres et l'importance du cours commercial.

En somme, nous nous trouvons devant une étude fort bien campée, intéressante et pouvant être utile à la fois au chercheur scientifique et à l'amateur d'histoire des institutions d'éducation classique et d'histoire régionale. L'auteur a su situer son étude dans l'historiographie contemporaine et son apport à la compréhension de l'histoire du Québec est tangible. Il enrichit l'histoire des collèges classiques en nous fournissant une monographie supplémentaire dans ce domaine, qui comprend une étude du cours commercial. L'imbrication et les objectifs précis d'une institution d'enseignement secondaire régionale sont bien abordés. Étant donné le caractère assez poussé de l'historiographie, du cadre théorique et des statistiques, l'amateur sera probablement plus attiré par les deux premières parties, la deuxième surtout. Mentionnons que, dans la première partie, la section sur la mutation socioreligieuse à Mont-Laurier et dans les Laurentides nous apparaît un peu mince et aurait eu avantage à être approfondie. Quant à la deuxième partie, le découpage en décennies (ou à peu près) de même que le choix de thèmes dont on ne retrouve pas toujours la continuité d'une période à l'autre rendent la lecture parfois répétitive et un peu fastidieuse. En définitive, toutefois, le contenu significatif de l'ensemble de l'ouvrage surpasse ces inconvénients mineurs. 